\title{
Proceeding
}

Supplementary Issue: Autumn Conferences of Sports Science. Costa Blanca Sports Science Events, 18-19 December 2020. Alicante, Spain.

\section{Verification of reliability of measurement systems for swimming analysis}

\author{
MICHAELA BÁTOROVÁ1 \\ ${ }^{1}$ Centre of Sports, Brno University of Technology, Brno, Czech Republic \\ ${ }^{2}$ Faculty of Physical Culture, Palacký University Olomouc, Olomouc, Czech Republic
}

\begin{abstract}
Sophisticated technologies and measuring devices are increasingly being used for the analysis of performance and swimming techniques. At BUT, the Tachograph II and Swimming Inertial Measure Unit (SwIMU) measuring systems were developed for swimming analysis. To evaluate the measured data, the SwimDataViewer software was developed for both measuring systems. To verify the reliability of the Tachograph, 34 race swimmers were measured (15 F, $19 \mathrm{M})$, and 28 race swimmers (16 $\mathrm{M}, 12 \mathrm{~F})$ were measured with SwIMU. All swimmers swam a 6x25m front crawl. Pullout and the first three and last two strokes of the arms were not included in the data evaluation. The intraclass correlation coefficient (ICC) was used to verify the reliability of the monitored parameters. The values of measured parameters in the front crawl for Tachograph (SwIMU) were ICC $=0.992$ (0.991) for the average value of speed, ICC $=0.958(0.960)$ for efficiency, ICC $=0.971(0.988)$ for the frequency of the arms, and finally, ICC $=0.978(0.992)$ for the frequency of the legs. The ICC coefficient for all monitored parameters in both devices showed a high degree of reliability. From this perspective, both devices are suitable for use in coaching and research practice.

Keywords: Swimming technique; Performance; Swimming analysis; Inertial measure unit; Reliability; Speed.

\section{Cite this article as:}

Bátorová, M., Št’astný, J., Elfmark, M., \& Janura, M. (2021). Verification of reliability of measurement systems for swimming analysis. Journal of Human Sport and Exercise, 16(2proc), S671-S677. doi:https://doi.org/10.14198/ihse.2021.16.Proc2.52
\end{abstract}

Corresponding author. Centre of Sports, Brno University of Technology, Technická 2896/2, 61669 Brno, Czech Republic. https://orcid.org/0000-0002-6011-3694

E-mail: michaela.batorova@vut.cz

Abstract submitted to: Autumn Conferences of Sports Science. Costa Blanca Sports Science Events, 18-19 December 2020. Alicante, Spain.

JOURNAL OF HUMAN SPORT \& EXERCISE ISSN 1988-5202

(c) Faculty of Education. University of Alicante

doi:10.14198/jhse.2021.16.Proc2.52 


\section{INTRODUCTION}

The use of modern technologies for motion analysis is a necessity nowadays, including swimming. In the past, measuring devices were based on tachometers and dynamometers such as Speedometer (Swim Speedo-meter; Swimsportec, Hildesheim, Germany), Torrent E-Rack (The Torrent ${ }^{\mathrm{TM}}$ E-Rack ${ }^{\mathrm{TM}}$ Electronic Swim Power Trainer ${ }^{\mathrm{TM}}$, TriLabs, California, USA) or Tachograph (Motyčka, Štastný, Lepková, Bátorová \& Pašek, 2013). Their use is currently less frequent, but even today there is still some development in this area.

Currently, the method of measuring the movement of swimmers using inertial sensors is perceived to be more effective than tachometric devices, mainly due to the possibility of a more detailed analysis of movement and measurement in three axes. Several studies have addressed the accuracy of IMU-based measurement methods (Marinho et al., 2019; Stamm et al., 2013; Dadashi et al., 2012).

Accuracy studies point to the main advantages of IMU swimming measurements: simple and fast preparation of measurements including calibration, less time-consuming processing of measured data with the possibility of automation using various algorithms, and the possibility of more detailed recording and analysis of motion in three axes. On the other hand, the studies pointed to problems related to wireless communication in the aquatic environment that complicate the analysis of real-time data, and the need for high-quality attachment of sensors to the swimmer's body (Marinho et al., 2019; Stamm et al., 2013; Dadashi et al., 2012).

Several research groups are involved in the measurement and analysis of swimming using an IMU. For example, Dadashi et al. (2013) performed measurements using an accelerometer to record the acceleration of swimming along the entire length of the pool. Puel, Seifert and Hellard (2014) developed the Ciren prototype, which is based on the NANO MEMSense inertial measuring unit, consisting of a combined triaxial accelerometer, magnetometer and gyroscope and an integrated control unit that records data at a frequency of $150 \mathrm{~Hz}$. To verify the reliability of IMU measurements, the authors used a speedometer and a multicamera system.

At the Brno University of Technology (BUT), the tachometric measuring device Tachograph II and an accelerometric measuring device Swimming Inertial Measuring Unit (SwIMU) were developed for the analysis of swimming technique and swimmer's performance, both with synchronous video recording. The aim of our research was to verify the reliability of both devices when measuring in the aquatic environment, to determine the level of accuracy when measuring in real conditions.

\section{METHODOLOGY}

\section{Measuring systems}

Tachograph II

Tachograph II was developed at the Brno University of Technology (BUT) to measure the speed of a swimmer's linear movement in an aquatic environment. Tachographs convert linear motion into rotary motion, which is converted to an electrical signal. The sensing of the swimming speed is performed by optoelectronic incremental rotary sensors, which sense the unwinding speed of the cable spool with a frequency of 1000 $\mathrm{Hz}$. This device consists of two instruments mounted on the front and back of the pool. A supporting cable with a predetermined preload is strung above the pool, along which the pulley suspension moves from the tachograph 1 to the tachograph 2. The ends of the Tachograph cables are connected to the pulley (from each side). The swimmer is attached to the pulley by a thin cable attached to a belt. While swimming forward, the swimmer unwinds the cable from Tachograph 1, while Tachograph 2 winds the cable so that the second 
(measuring) cable does not sag (Figure 1). When swimming back, the functions of the tachographs are swapped. The speed of swimming is always measured by the tachograph that the cable is unwound from.

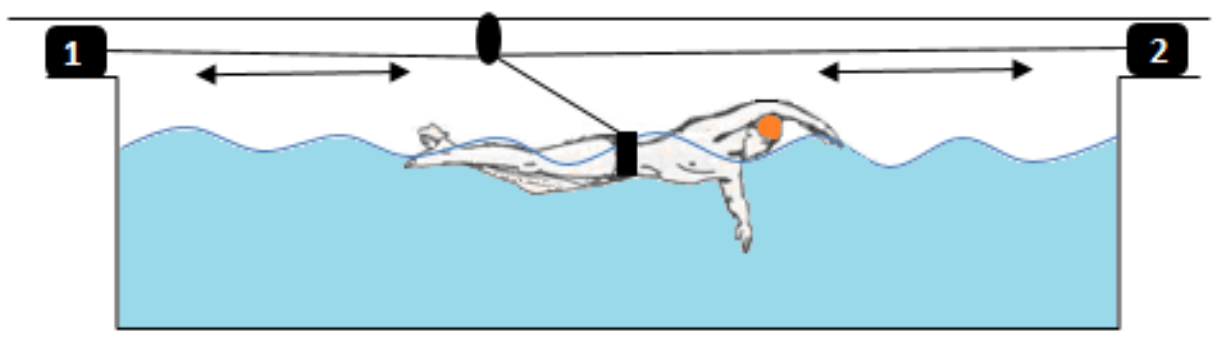

Figure 1. Schematic representation of the Tachograph II measuring system.

The measured speed data from Tachograph II together with the recording of two static underwater video cameras and one moving camera recording the swimmer from the side are processed and synchronized in the software SwimDataViewer 5.2.2. This system allows us to measure the speed of a swimmer on a track of any length (in pools with a length of 25 and $50 \mathrm{~m}$ ) in the x-axis, but does not allow us to measure turns, starts or clearly determine in which axis the speed of the swimmer increases.

Therefore, the development of the SwIMU measuring device was started at BUT with the aim of creating a device that enables the measurement of acceleration and speed together with synchronous video recording, without the need to attach swimmers to the device with ropes and limit their movement. At the same time, the device should enable the measurement of acceleration and speed more accurately, and in all three axes.

\section{Swimming Inertial Measuring Unit}

The SwIMU system is compatible with the original tachographic system (Tachograph II), so measurements can be performed simultaneously on both systems, including the synchronized recording of underwater video cameras. However, the new system allows the measurement of acceleration and to obtain speed from that data in all three axes, including starts and turns, which is not possible with Tachograph II. Data measured using the IMU are processed in a new version of our software SwimDataViewer 5.2.2. The software and hardware solution of SwIMU was developed according to the needs of both coaching and research, and its further development is still in progress.

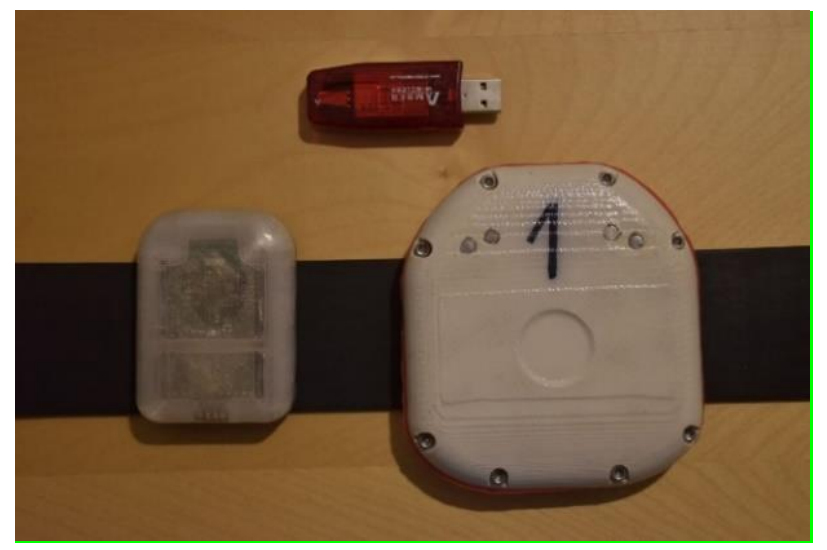

Figure 2. Larger version of IMU 1 (right) and a new smaller reduced version of IMU (left) and USB RF receiver (top). 
The SwIMU consists of an inertial measuring unit that contains a triaxial MEMS accelerometer and a gyroscope (MPU-6000, InvenSense Inc, U.S.A.), a microcontroller (STM32L4, STMicroelectronics, Switzerland), internal memory and a communications interface in a watertight housing (measurement was performed with a larger version of the IMU - Figure 2 - right). During the measurement, data are stored in flash memory and after the measurement, data are downloaded to the computer, where they are synchronized with the video recording in the software.

\section{Research set}

To verify the reliability of Tachograph $I, 19$ men were measured $(m=71.4 \pm 12.8 \mathrm{~kg}, \mathrm{~h}=182.2 \pm 8.6 \mathrm{~cm}$, age $=17.6 \pm 3.2$ years $)$ and 15 women $(n=15 \mathrm{~m}=61.5 \pm 6.4 \mathrm{~kg}, \mathrm{~h}=172.8 \pm 5.5 \mathrm{~cm}$, age $=16.3 \pm 2.1$ years) and to verify the SwIMU, $16 \mathrm{men}(\mathrm{m}=77.5 \pm 11.6 \mathrm{~kg}, \mathrm{~h}=183.9 \pm 10.5 \mathrm{~cm}$, age $=19.6 \pm 3.9$ years $)$ and 12 women $(\mathrm{m}=59.6 \pm 4.7 \mathrm{~kg}, \mathrm{~h}=170.1 \pm 5.7 \mathrm{~cm}$, age $=18.3 \pm 2.3$ years $)$. All participants were competitive swimmers from the junior and senior national teams and swimmers from sports grammar schools. Swimmers had no health restrictions and were not selected for their swimming specialization or performance.

All research participants gave their written informed consent to the processing of personal data and measured data. The research was approved by the ethics committee of the Center for Sports Activities of the Brno University of Technology.

\section{Realization of measurements}

The measurement occurred in the middle lane of a three-lane pool with a length of 25 meters and a water temperature of about $27^{\circ} \mathrm{C}$. In order not to disturb the video recording by further swirling of the water, only the measured swimmer was in the pool at the time of the measurement.

Each swimmer had the opportunity to swim and stretch as needed before the measurement. Subsequently, the SwIMU device in a watertight case or a belt with a cable from the Tachograph was attached to the swimmer on the lower part of the back (at the level of the third lumbar vertebra). The heart rate (HR) of each swimmer was measured before the first measured section and during repeated measurements using a Polar chest belt sports tester (RCX5, Polar, Finland). The participants had the opportunity to swim freely for a short time $(25 \mathrm{~m})$ between the individual swimming sections and then rested at the edge of the pool. All swimmers only started the repeated measured section when their HR dropped to at least the starting level of HR that they had before the first measured section. With this approach, we tried to eliminate the effect of fatigue between individual swimming sections as much as possible.

Each swimmer started from the water (Tachograph does not allow a dive start) and swam six 25-meter sections with the maximum effort in freestyle.

After pushing off the wall, there was an underwater pull-out (movement by butterfly kick) with a transition to freestyle legs and combined freestyle swimming. Each swimmer had an individual pull-out (according to the swimming rules, a maximum distance of $15 \mathrm{~m}$ underwater was set). After a touchdown on the opposite side of the pool, the swimmer swam back freely (without measuring). Subsequently, the swimmer left the pool and the radio signal was used to transfer data from SwIMU to a PC. Data from cameras and tachographs were stored online on a PC.

\section{Data processing and measured parameters}

204 measurements of 25-meter sections were performed. During the evaluation in SwimDataViewer 5.2.2, each section was divided into the pull-out part and the combined freestyle. To verify the reliability of SwIMU 
and Tachograph, we only evaluated the section of the freestyle without the first three and last two freestyle strokes, as Tachograph II does not allow the measurement of pull-out. By not evaluating the first 3 and last 2 freestyle strokes, we made sure that the evaluated technique was stable and not affected by either the pullout or the final touch. From the data contained in the selected time period (swimming interval), the software calculated the efficiency of swimming technique (the most effective swimming technique is one that has the smallest possible speed drops within one swimming cycle and over the whole track, ideally at the highest average speed), average swimming speed $\left(\mathrm{m} \cdot \mathrm{s}^{-1}\right)$ and its coefficient of variation, standard deviation $\left(\mathrm{m} \cdot \mathrm{s}^{-1}\right)$ and variance (Motyčka et al., 2013; Štastný et al., 2016). The frequency of the arms and legs was taken manually from the video recording and their average frequency was automatically calculated by the software.

Data analyses were performed using the STATISTICA (version 12.0, StatSoft, Inc., Tulsa, OK, USA) software. To determine the degree of reliability of the Tachograph II and SwIMU measuring devices, the testretest method was used, and subsequently the intraclass correlation (ICC) values of the measured parameters with indicative limits were calculated. We used the following thresholds to assess reliability: ICC $\leq 0.75$ Poor to moderate reliability, ICC $>0.75$ Good reliability, ICC $\geq 0.90$ High reliability, required for clinical applications to ensure the valid interpretation of findings (Innes \& Strake 1999).

\section{RESULTS}

In order to claim that the instruments are reliable, the ICC values of the measured and evaluated parameters must be above 0.9, which was met for all parameters (Figure 3). All parameter values were higher than 0.958 for both Tachograph II and SwIMU. Based on the results, we can say that both Tachograph and SwIMU demonstrate a high degree of reliability.

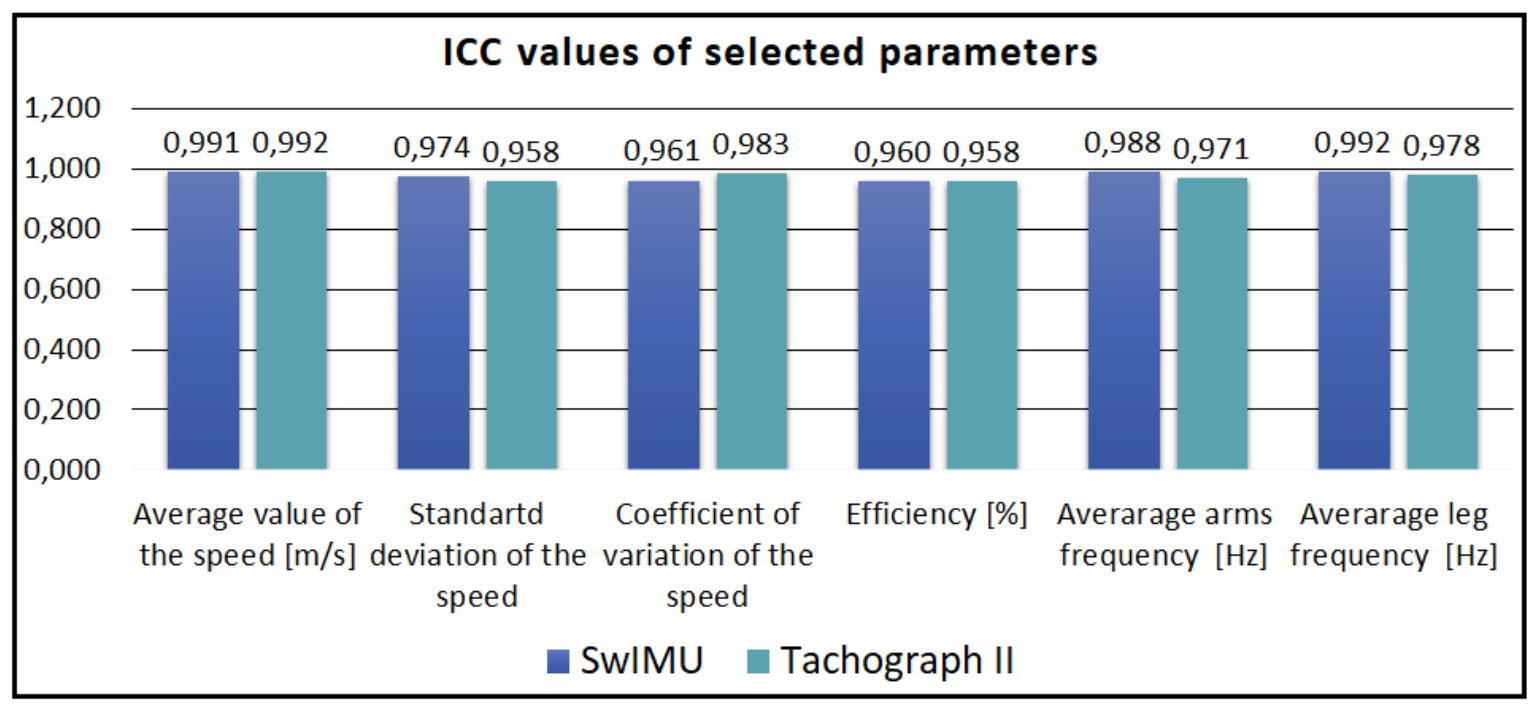

Figure 3. Graphical representation of ICC correlation coefficients for parameters measured by SwIMU and Tachograph II.

\section{DISCUSSION}

Both Tachograph II and SwIMU are measuring systems with a high level of reliability for all measured parameters. Despite the fact that Tachograph II directly measures the speed of the swimmer and SwIMU 
measures the acceleration and the swimming speed is calculated from it, the reliability of speed measurement for both methods is above 0.99 . We attribute the different ICC results for the frequency of the legs to the human factor, as the frequency of legs and arms is manually read and further processed by the software from the camera recording; however, the ICC value is still above 0.97 .

Despite the high degree of reliability of both methods, it is important to work with factors and limits of research that influence the accuracy of measurements. These factors influencing the accuracy of the measurement included the fact that the effect of swimmer fatigue cannot be completely eliminated during repeated measurements. Pilot measurements were performed, where swimmers swam ten 25-meter sections, which, however, had a very pronounced effect of fatigue (large differences in speed between the first and second half of the measurement), so the number of repetitions was reduced to eight and then to six. To reduce the effect of fatigue, we included HR measurements, where swimmers were not allowed to start swimming the next measured section before their HR dropped back to the value they had before swimming the first measured section. Another factor influencing the swimmer's speed, and thus the quality of measurement, was stress. For some participants, the measurement may have caused stress that could affect their performance and/or its stability. Last but not least, the limits of the research include the fact that swimmers were instructed to swim six sections of 25 meters at maximum speed, but it is not entirely possible to ensure that swimmers follow these instructions and actually swim their maximum intensity in each measured section, e.g., that they do not start swimming slower, or they do not start accelerating at the end of the measurement, etc.

Accelerometers undergo drifts (Stamm et al., 2013; Dadashi et al., 2012), which reduce the accuracy of measurements and must be compensated. The method of mounting the accelerometer to the swimmer's body and the larger dimensions of the IMU may have a greater effect on the accuracy of the measurement due to the greater hydrodynamic resistance (Marinho et al., 2019). That is why we keep working to reduce the dimensions (Figure 2) and optimize the attachment of the SwIMU to the belt to prevent unwanted movements due to the flowing water between the swimmer's body and the measuring device.

Among the mechanical factors influencing the accuracy of Tachograph II measurements, we can include the swing of the tachograph support cable. Furthermore, Tachograph II is in principle only able to measure speed in the $x$-axis, so it is not possible to clearly evaluate in which axis the speed increases. Additionally, Tachograph II does not allow the measurement of starting dives, pull-outs, or turns, which is important for coaching. Tachometric devices such as the Speedometer measure pull-outs and combined swimming, but also without starting dives and turns (Barbosa et al., 2013).

\section{CONCLUSION}

The ICC coefficient for all the monitored parameters in both devices showed a high degree of reliability, above 0.9. From this point of view, both Tachograph II and SwIMU are suitable devices for use in coaching and research with regard to measurement requirements.

\section{REFERENCES}

Barbosa, T., Morouço, P. G., Jesus, S., Feitosa, W., Costa, M., Marinho, D., Silva, A., \& Garrido, N. (2013). The Interaction Between Intra-Cyclic Variation of the Velocity and Mean Swimming Velocity in Young Competitive Swimmers. International Journal Of Sports Medicine, 34(02), 123-130. https://doi.org/10.1055/s-0032-1312582 
Dadashi, F., Crettenand, F., Millet, G. P., \& Aminian, K. (2012). Front-Crawl Instantaneous Velocity Estimation Using a Wearable Inertial Measurement Unit. Sensors, 12(10), 12927-12939. https://doi.org/10.3390/s121012927

Dadashi, F., Millet, G. P., \& Aminian, K. (2013). Inertial measurement unit and biomechanical analysis of swimming: An update. Schweizerische Zeitschrift Fur Sportmedizin Und Sporttraumatologie, 61(3), 28-33.

Innes, E., \& Straker, L. (1999). Reliability of work-related assessments. Work (Reading, Mass.), 13(2), 107-124.

Marinho, D. A., Neiva, H. P., \& Morais, J. E. (2019). The Use of Wearable Sensors in Human Movement Analysis in Non-Swimming Aquatic Activities: A Systematic Review. International Journal Of Environmental Research And Public Health, 16(24). https://doi.org/10.3390/ijerph16245067

Motyčka, J., Št’astný, J., Lepková, H., Bátorová, M., \& Pašek, M. (2013). Kinematic and dynamic analyses of swimming. In In Sport and Quality of Life. (pp. 14-24). Masarykova universita.

Puel, F., Seifert, L., \& Hellard, P. (2014). Validation of an inertial measurement unit for the determination of the longitudinal speed of a swimmer. In Biomechanics and Medicine in Swimming XII.

StatSoft, Inc. (2013). STATISTICA (data analysis software system), version 12. www.statsoft.com

Stamm, A., James, D. A., \& Thiel, D. V. (2013). Velocity profiling using inertial sensors for freestyle swimming. Sports Engineering, 16(1), 1-11. https://doi.org/10.1007/s12283-012-0107-6

Št’astný, J., Motyčka, J., Bátorová, M., \& Pašek, M. (2016). Changes in speed and efficiency in the front crawl swimming technique at $100 \mathrm{~m}$ track. Journal Of Human Sport And Exercise, 11(Proc1), 168175. https://doi.org/10.14198//hse.2016.11.Proc1.07

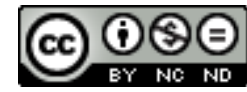

This work is licensed under a Attribution-NonCommercial-NoDerivatives 4.0 International (CC BY-NC-ND 4.0). 